\title{
IT-Backsourcing - Empirische Erkenntnisse und Handlungsempfehlungen für eine erfolgreiche Re-Integration der IT-Serviceerbringung
}

\author{
Benedikt von Bary $•$ Markus Westner $\cdot$ Susanne Strahringer $\mathbb{D}$
}

Eingegangen: 22. Mai 2019 / Angenommen: 24. Januar 2020 / Online publiziert: 5. Februar 2020

(C) Der/die Autor(en) 2020

Zusammenfassung IT-Backsourcing bezeichnet die Re-Integration vormals outgesourcter IT-Services zurück zum auslagernden Unternehmen. Die vorliegende Studie stellt die Ergebnisse einer Umfrage unter IT-Managern zu Einflussfaktoren von IT-Backsourcing-Entscheidungen vor. Es lässt sich feststellen, dass IT-Backsourcing von Unternehmen in unterschiedlichen Branchen und für eine Vielzahl an unterschiedlichen IT-Services, wie z. B. Applikationsentwicklung oder InfrastrukturLeistungen, durchgeführt wird. Hauptgründe für die Entscheidung für ein IT-Backsourcing sind mangelnde Qualität, hohe Kosten sowie eine schlechte Beziehung zum IT-Dienstleister. Die Ergebnisse der Umfrage zeigen, dass diejenigen Unternehmen, die sich für ein Backsourcing entschieden haben, nach der Entscheidung insgesamt zufriedener waren als diejenigen, die in einer Outsourcing-Beziehung geblieben sind. Zudem werden verschiedene Handlungsempfehlungen für IT-Manager aufgezeigt. Zur Ausarbeitung einer zukunftsorientieren IT-Sourcing-Strategie empfiehlt es sich, eine service-spezifische Sourcing-Strategie zu verfolgen und entsprechend spezialisierte Dienstleister einzusetzen. Im Falle einer Entscheidung für ein IT-Backsourcing sollten verschiedene Erfolgsfaktoren beachtetet werden, wie z.B. eine frühzeitige Neubesetzung von Schlüsselstellen sowie ein lückenloser Wissenstransfer.

Schlüsselwörter Backsourcing · Insourcing · IT-Sourcing · Re-Integration · ITService $\cdot$ Erfolgsfaktoren

\footnotetext{
B. von Bary $\cdot$ S. Strahringer $(\bowtie)$

Wirtschaftsinformatik, insb. Informationssysteme in Industrie und Handel, Technische Universität Dresden, Helmholtzstr. 10, 01069 Dresden, Deutschland

E-Mail: Susanne.Strahringer@tu-dresden.de

M. Westner

Fakultät Informatik und Mathematik, Ostbayerische Technische Hochschule Regensburg,

Galgenbergstr. 32, 93053 Regensburg, Deutschland
} 


\title{
IT Backsourcing - Empirical Findings and Recommendations for Bringing IT Service Delivery Back
}

\begin{abstract}
IT backsourcing describes the re-integration of previously outsourced IT services back to the outsourcing company. This paper presents the results of a survey with IT managers about factors influencing IT backsourcing decisions. The survey confirms that IT backsourcing is undertaken by companies from different industries and for a variety of different IT services, such as application development or infrastructure services. The main reasons for deciding in favor of IT backsourcing are poor quality, high costs and a poor relationship with the IT service provider. Further, the results of the survey show that after the decision, those companies that opted for backsourcing were generally more satisfied than those that remained in an outsourcing relationship. In addition, this paper provides several recommendations for action for IT managers. To develop a future-oriented IT sourcing strategy, it is recommended to pursue a service-specific sourcing strategy and to use multiple, specialized service providers based on their respective strengths. After deciding for IT backsourcing, managers should consider different success factors, such as the early recruitment of personnel for key positions and a seamless knowledge transfer.
\end{abstract}

Keywords Backsourcing · Insourcing $\cdot$ IT-Sourcing $\cdot$ IT Service $\cdot$ Success Factors

\section{Backsourcing vormals ausgelagerter IT-Services}

Outsourcing-Partner sind nie so flexibel, wie es ein internes Team in der neuen, agilen Welt sein kann. Outsourcing-Partner können nur langsam auf Änderungen reagieren. (Zitat Studienteilnehmer)

Dieses Zitat eines Teilnehmers an einer kürzlich von den Autoren dieses Artikels durchgeführten Studie zum Thema „Einflussfaktoren von IT-Backsourcing-

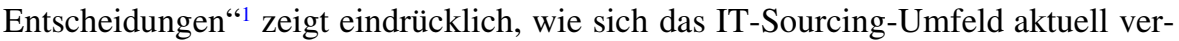
ändert und welche Herausforderungen dadurch entstehen können. Durch den zunehmenden Trend zur Digitalisierung in nahezu allen Industrien bekommt die IT im Unternehmen eine immer größere, strategisch wichtigere Bedeutung. So wird eine schnelle, innovative Unternehmens-IT zunehmend als Grundvoraussetzung gesehen, um zukunftsfähige, digitale Geschäftsmodelle erfolgreich am Markt anbieten zu können. Entsprechend stehen die jeweils verantwortlichen IT-Abteilungen vor der Herausforderung, die Bestandssysteme sowie bestehende Beziehungen zu ITDienstleistern an neue Anforderungen anzupassen. Hierfür existieren eine Vielzahl von Ansätzen, beispielsweise die Schaffung einer „Two Speed-IT“ zur schnellen Umsetzung von digitalen Angeboten, gepaart mit einer langfristigen Erneuerung der bestehenden Systeme oder alternativ auch der Schaffung von standardisierten Schnittstellen zwischen unternehmenseigenen Applikationen für eine schrittweise

\footnotetext{
1 Weitere Details zu Ablauf, Zielsetzungen und den Teilnehmern der Umfrage werden in Kap. 3 vorgestellt.
} 
Erneuerung der Bestandssysteme (Ketterer et al. 2016). Eine weitere Möglichkeit besteht darin, statt auf externe IT-Dienstleister verstärkt auf eigene Ressourcen zu setzen und entsprechende interne IT-Fähigkeiten langfristig (wieder) aufzubauen. Dieses Konzept wird meist als IT-Backsourcing oder auch Insourcing bezeichnet (Bary et al. 2018b).

Während IT-Outsourcing in der Forschung in den letzten Jahren viel thematisiert wurde, ist der Bereich des IT-Backsourcings - trotz seiner zunehmenden Relevanz im Kontext der Digitalisierung - weitaus weniger untersucht worden (Bary und Westner 2018). Entsprechend zielt die vorliegende Studie unter IT-Managern darauf ab, die Einflussfaktoren auf IT-Backsourcing-Entscheidungen sowie die Zufriedenheit mit dem IT-Sourcing-Arrangement nach der getroffenen Entscheidung genauer zu beleuchten. Entsprechend ergeben sich zwei zentrale Untersuchungsfragen:

1. Welche Gründe veranlassen Unternehmen dazu, (Teile) ihre(r) outgesourcten ITServiceerbringung wieder zurück in das eigene Unternehmen zu holen?

2. Was sind die Ergebnisse von IT-Backsourcing-Entscheidungen, vor allem hinsichtlich der Zufriedenheit mit der geänderten IT-Serviceerbringung?

Im weiteren Verlauf werden zuerst wichtige Grundlagen des IT-Backsourcings aus der bestehenden wissenschaftlichen Literatur abgeleitet und verschiedene Ausgestaltungsmöglichkeiten von IT-Backsourcing-Vorgängen beschrieben. Im Anschluss daran wird näher auf die Methodik der Umfrage eingegangen, bevor dann im Anschluss die Ergebnisse der Studie vorgestellt werden. Hierbei wird besonders auf unterschiedliche Ansätze und Gründe für ein IT-Backsourcing sowie die Resultate der Entscheidung eingegangen. Abschließend werden konkrete Handlungsempfehlungen für eine zukunftsorientierte IT-Sourcing-Strategie vorgestellt und konkrete Erfolgsfaktoren für die Umsetzung eines erfolgreichen IT-Backsourcings beschrieben.

\section{Grundlagen des IT-Backsourcings}

\subsection{Begriffsdefinition und Abgrenzung}

Der Begriff IT-Backsourcing beschreibt das Zurückholen von zuvor ausgelagerten IT-Services. Er wurde in der wissenschaftlichen Literatur als erstes von Hirschheim und Lacity (1998) und Lacity und Willcocks (2000) eingeführt. Das Ziel von ITBacksourcing ist es, dass das jeweilige Unternehmen im Endzustand die vormals ausgelagerten IT-Services wieder selbstständig erbringen kann.

Ein wesentliches Merkmal von Backsourcing ist, dass das Eigentum an allen benötigten Ressourcen und damit auch die Verantwortung für die Erfüllung der betroffenen IT-Services zurück an das outsourcende Unternehmen geht (Nujen et al. 2015). Dabei spielt der eigentliche Ort der Leistungserfüllung eine nachgelagerte Rolle. Ein räumliches Zurückholen ist nicht immer notwendig, denn die Services können auch in unterschiedlichen Ländern bzw. Regionen erbracht werden. Dies unterscheidet Backsourcing von verwandten Begriffen, die stärker auf eine Ände- 
rung des Erfüllungsortes abzielen, wie z. B. Reshoring, Backshoring oder Relocating (Bary und Westner 2018).

Neben dem Begriff Backsourcing, der in der Wissenschaft der gebräuchlichere Begriff ist, werden in der Praxis häufig auch die Begriffe „Insourcing“ oder, im englischen Sprachraum, auch ,Back in-house“ synonym verwendet (Bary et al. 2018b). Dabei lässt sich beobachten, dass der Begriff „Insourcing“ in der Praxis sowohl für den finalen Zustand der internen Bereitstellung der IT-Services als auch für den Vorgang des Zurückholens benutzt wird. Im Sinne der begrifflichen Trennschärfe wird in diesem Beitrag der Begriff „Backsourcing“ verwendet, um das eigentliche Zurückholen von IT-Services zu beschreiben. „Insourcing“ dagegen beschreibt die interne Bereitstellung von IT-Services.

\subsection{Unterschiedliche Ausgestaltungsmöglichkeiten für IT-Backsourcing}

IT-Backsourcing erfolgt zwangsläufig immer nach einer vorausgegangenen Phase des Outsourcings der jeweiligen IT-Services an einen externen Dienstleister (Thakur-Wernz 2019). Abhängig von der Ausgestaltung dieser ursprünglichen Outsourcing-Beziehung gibt es verschiedene Kombinationen, wie ein Unternehmen seine ITServices zurück holen kann (Bary 2018). Beispielsweise könnte sich der Standort des Outsourcing-Dienstleisters im selben Land (,onshoring“), in derselben Region (,nearshoring“) oder in einer anderen Region (,offshoring“) als der des outsourcenden Unternehmens befinden (Strasser und Westner 2015). Oder das Unternehmen könnte entweder seine gesamte IT-Abteilung oder nur die Bereitstellung von ausgewählten IT-Services ausgelagert haben (Dibbern et al. 2004). Diese Vielzahl von möglichen Outsourcing-Kombinationen erhöht entsprechend auch die Komplexität der Backsourcing-Entscheidung.

Während in jedem Fall ein Eigentümerwechsel vom bisherigen IT-Dienstleister hin zum Mutterunternehmen erfolgt (Nujen et al. 2015), kann sich die konkrete Ausgestaltung eines jeden Backsourcing-Vorgangs dagegen unterscheiden. Dadurch ergeben sich verschiedene Dimensionen, um Backsourcing-Fälle zu vergleichen und zu klassifizieren (Bary 2018).

Zuerst lassen sich zwei Dimensionen unterscheiden, die die Backsourcing-Situation des Unternehmens betrachten. Hinsichtlich des Zielstandorts der Leistungserbringung nach dem Backsourcing kann entweder kein Wechsel, ein Wechsel in das Heimatland des Unternehmens oder auch ein Wechsel weiter weg vom Standort des Unternehmens, wie z.B. nach Indien, erfolgen (Oshri et al. 2015). Daneben kann auch entlang des bisherigen Zustands der IT-Abteilung unterschieden werden (Bary 2018). So könnte die bisherige IT-Abteilung, die sog. ,retained organization“ des Unternehmens, lediglich mit dem Lieferantenmanagement betraut gewesen sein, einen abgegrenzten Funktionsumfang bereit gestellt haben oder parallel für den vollen Funktionsumfang zuständig gewesen sein und nur einen kleinen Teil outgesourct haben.

Neben diesen beiden Dimensionen lassen sich noch zwei weitere Dimensionen unterscheiden, die eher auf die Backsourcing-Durchführung abzielen: So lassen sich unterschiedliche Strategien zum Aufbau von internen Fähigkeiten unterscheiden, je nachdem, ob das Unternehmen die IT-Abteilung und entsprechende Ressourcen 


\begin{tabular}{|c|c|c|c|c|}
\hline Zielstandort & $\begin{array}{l}\text { Kein Wechsel des } \\
\text { Standortes für die } \\
\text { Erbringung der IT- } \\
\text { Services }\end{array}$ & $\begin{array}{l}\text { Wechsel zu einem } \\
\text { Standort im gleichen } \\
\text { Land wie das } \\
\text { Mutterunternehmen }\end{array}$ & $\begin{array}{l}\text { Wechsel an einen } \\
\text { near-/offshore } \\
\text { Standort }\end{array}$ & \multirow{2}{*}{$\begin{array}{l}\text { Back- } \\
\text { sourcing- } \\
\text { Situation }\end{array}$} \\
\hline $\begin{array}{l}\text { Bisheriger } \\
\text { Zustand der } \\
\text { IT-Abteilung }\end{array}$ & $\begin{array}{l}\text { Nur Lieferanten- } \\
\text { management, ohne } \\
\text { eigene Entwicklungs- } \\
\text { kapazitäten }\end{array}$ & $\begin{array}{l}\text { Eingeschränktes } \\
\text { Aufgabenspektrum der } \\
\text { int. IT-Organisation } \\
\text { (z.B. Fokus auf } \\
\text { bestimmte Aufgaben) }\end{array}$ & $\begin{array}{l}\text { Umfassendes } \\
\text { Aufgabenspektrum } \\
\text { der internen IT- } \\
\text { Organisation }\end{array}$ & \\
\hline $\begin{array}{l}\text { Strategie zum } \\
\text { Aufbau von } \\
\text { internen } \\
\text { Fähigkeiten }\end{array}$ & $\begin{array}{l}\text { Ausschließlich } \\
\text { organischer Aufbau, } \\
\text { z.B. durch Einstellung } \\
\text { neuer Mitarbeiter }\end{array}$ & $\begin{array}{l}\text { Kombination aus } \\
\text { organischem and } \\
\text { anorganischem Aufbau }\end{array}$ & $\begin{array}{l}\text { Ausschließlich } \\
\text { anorganischer } \\
\text { Aufbau, z.B. durch } \\
\text { Übernahmen }\end{array}$ & \multirow{2}{*}{$\begin{array}{l}\text { Back- } \\
\text { sourcing- } \\
\text { Durch- } \\
\text { führung }\end{array}$} \\
\hline $\begin{array}{l}\text { Externe } \\
\text { Unterstützung } \\
\text { während des } \\
\text { Backsourcings }\end{array}$ & $\begin{array}{l}\text { Keine externe } \\
\text { Unterstuitzung } \\
\text { (z.B., durch Berater) }\end{array}$ & $\begin{array}{l}\text { Externe Unterstützung } \\
\text { während der } \\
\text { Backsourcing- } \\
\text { Durchführung }\end{array}$ & $\begin{array}{l}\text { Temporäre } \\
\text { Unterstützung für } \\
\text { die Bereitstellung } \\
\text { der IT-Services }\end{array}$ & \\
\hline
\end{tabular}

Abb. 1 Dimensionen eines Backsourcing-Vorgangs mit beispielhafter Einordnung des Backsourcings von Thyssenkrupp. ${ }^{\text {a}}$ Während des Outsourcings, vor dem Backsourcing der IT-Services. (In Anlehnung an Bary (2018))

ausschließlich organisch (z.B. durch Einstellung neuer Mitarbeiter), ausschließlich anorganisch (z.B. durch Übernahmen) oder in einer Mischform aufbaut (Bhagwatwar et al. 2011; Butler et al. 2011). Abschließend lässt sich auch unterscheiden, ob das Unternehmen externe Unterstützung während des Backsourcings in Anspruch nimmt, z. B. während der Backsourcing-Durchführung oder darüber hinaus eine temporäre Unterstützung bei der Bereitstellung der IT-Services durch eine dritte Partei (Bary 2018). Abb. 1 zeigt die vier vorgestellten Dimensionen mit ihren möglichen Ausprägungen.

Exemplarisch ist hier der Backsourcing-Fall von Thyssenkrupp zum Ende des Jahres 2017 durch graue Hinterlegung eingeordnet. Der 2014 abgeschlossene Siebenjahresvertrag mit dem IT-Dienstleister T-Systems hatte das Ziel der IT-Konsolidierung, unter anderem durch den Aufbau zentraler, extern betriebener Rechenzentren (Heeg 2018). Gründe für das Backsourcing waren einerseits sowohl Unzufriedenheit über Kosten, Qualität und Zusammenarbeit, andererseits jedoch auch eine strategische Neuausrichtung mit dem Ziel, eine geringere Abhängigkeit von einem einzelnen Dienstleister und verstärktes Multi-Vendor-Sourcing zu erreichen (Nickel 2018). Aus den öffentlich verfügbaren Informationen lässt sich schließen, dass es durch das Backsourcing zunächst anscheinend zu keinem Wechsel des Standortes für die Serviceerbringung kam (Heeg 2018; Karabasz und Wocher 2018). Zudem hatte die IT-Abteilung auch während des Outsourcings noch einen eingeschränkten Funktionsumfang und war nicht allein mit dem Lieferantenmanagement beauftragt (Heeg 2018). Im Rahmen des Backsourcings wurden ca. 100 Mitarbeiter, die ursprünglich von Thyssenkrupp zu T-Systems gewechselt waren, wieder zurück zu Thyssenkrupp geholt (Karabasz und Wocher 2018). In Kombination mit einem zusätzlichen organischen Stellenaufbau ist hier von einer kombinierten anorganischen 
und organischen Strategie zum Aufbau von internen Fähigkeiten auszugehen. Über eine externe Unterstützung während der Backsourcing-Durchführung ließen sich keine Informationen finden, entsprechend wird angenommen, dass dies durch interne Ressourcen erfolgte.

Die konkrete Ausgestaltung sowohl des eigentlichen Backsourcing-Vorgangs als auch des finalen Insourcing-Zielzustands hängt einerseits stark von der Ausgangssituation des Unternehmens ab, lässt sich andererseits jedoch auch an die Zielsetzung des Unternehmens anpassen. Entsprechend werden im Kap. 5 konkrete praktische Handlungsempfehlungen vorgestellt, wie ein Unternehmen ein Backsourcing-Vorhaben erfolgreich umsetzen kann.

\section{Untersuchungsmethode}

Die vorliegende Studie verfolgt einen empirischen Forschungsansatz zur Beantwortung der eingangs vorgestellten Untersuchungsfragen. Die Datenerhebung erfolgte durch eine Online-Umfrage und hatte das Ziel, Einflussfaktoren von IT-Backsourcing-Entscheidungen sowie die Zufriedenheit mit dem Sourcing-Arrangement nach der Sourcing-Entscheidung zu untersuchen. Der zugrundeliegende Fragebogen orientiert sich an einem möglichen Backsourcing-Prozess: Zunächst wurde die Situation während des IT-Outsourcings abgefragt, z. B. die outgesourcten IT-Services, die Industrie oder auch die angestrebte Dauer der Outsourcing-Beziehung. Im Anschluss wurde genauer auf den Entscheidungszeitpunkt eingegangen und z. B. die von der Entscheidung betroffenen IT-Services oder die Entscheidungsgründe erfragt. Abschließend wurde dann auf die Situation nach der Entscheidung eingegangen und die Zufriedenheit mit der Leistungserbringung nach der Entscheidung adressiert. Zudem wurden weitere Kontextinformationen abgefragt, wie beispielsweise die Rolle des Teilnehmers im Zuge der Entscheidung, Herkunft und Branche des Unternehmens sowie die Art des betrachteten IT-Services.

Der erhobene Datensatz enthält bewusst auch Unternehmen, bei denen ein ITBacksourcing zwar diskutiert, aber letztendlich eine Entscheidung gegen ein ITBacksourcing getroffen wurde. Dies ermöglicht einen Vergleich der Zufriedenheit mit einer Entscheidung für IT-Backsourcing mit solchen Unternehmen, die sich für einen Verbleib in der IT-Outsourcing-Beziehung entschieden haben.

Die Umfrage richtete sich an IT-Manager mit Erfahrung in IT-Sourcing-Entscheidungen - insbesondere an solche, die bereits in Entscheidungen involviert waren, bei denen IT-Backsourcing eine der möglichen Entscheidungsoptionen war. Die Teilnehmer der Umfrage sollten entweder bei betroffenen Unternehmen, bei ITDienstleistern oder auch bei Beratungsunternehmen angestellt sein. Es wurden verschiedene Kanäle benutzt, um geeignete Teilnehmer zu erreichen. Am wichtigsten waren dabei die beiden Karrierenetzwerke LinkedIn und XING. In beiden Netzwerken wurde nach Profilen gesucht, die als Berufsbezeichnung „IT-Projektleiter“ oder ähnliches und zusätzlich die Schlüsselwörter „Insourcing“ oder/und „Backsourcing“ angegeben hatten. Die Auswertung für diesen Beitrag fokussiert sich auf Unternehmen im deutschsprachigen Raum. Zusätzlich wurden CIOs und CTOs von DAX-, MDAX-, und SDAX-Unternehmen direkt per E-Mail mit der Bitte angeschrieben, 
an der Studie teilzunehmen. Um noch weitere potentielle relevante Teilnehmer zu erreichen, wurde außerdem mit dem Outsourcing Verband ${ }^{2}$, einem der führenden ITOutsourcing-Netzwerke in Europa, zusammengearbeitet.

Die Umfrage wurde im Dezember 2018 gestartet und nach einer 4-monatigen Laufzeit Anfang April 2019 beendet. Insgesamt gingen 80 vollständige Antworten aus der DACH-Region ein. Aufgrund der Tatsache, dass wir potentielle Studienteilnehmer über verschiedene Kanäle wie Newsletter teils anonym kontaktiert haben, ist es uns nicht möglich, die tatsächliche Anzahl der erzielten Kontaktaufnahmen zu ermitteln. Entsprechend lässt sich keine Rücklaufquote berechnen.

\section{Ergebnisse der Umfrage zu Backsourcing-Erfahrungen von IT- Managern}

\section{1 Überblick über die Teilnehmer der Umfrage}

Von den Teilnehmern der Umfrage war ein Großteil (49; 64\%) der Befragten bei einem outsourcenden Unternehmen beschäftigt. Dagegen waren $21(27 \%)$ an der Entscheidung beteiligte Berater und $7(9 \%)$ bei einem IT-Dienstleister angestellt. Betrachtet man die Herkunft der Unternehmen (d.h. den Sitz der Unternehmenszentrale oder der relevanten Geschäftseinheit), die die Sourcing-Entscheidungen getroffen haben, wurden $87 \%$ (70) der Entscheidungen von Unternehmen in Deutschland, $10 \%$ (8) von Unternehmen in der Schweiz und 3\% (2) von Unternehmen in Österreich getätigt. Dies entspricht im Wesentlichen der nationalen Verteilung der Studienteilnehmer, die für die Umfrage kontaktiert wurden.

Die betrachteten Unternehmen waren größtenteils Großunternehmen (71; 90\%) mit einer Mitarbeiterzahl von über 250 und einem Umsatz von mehr als 50Mio. EUR. Hinsichtlich des Budgets der zugrunde liegenden Outsourcing-Verträge lässt
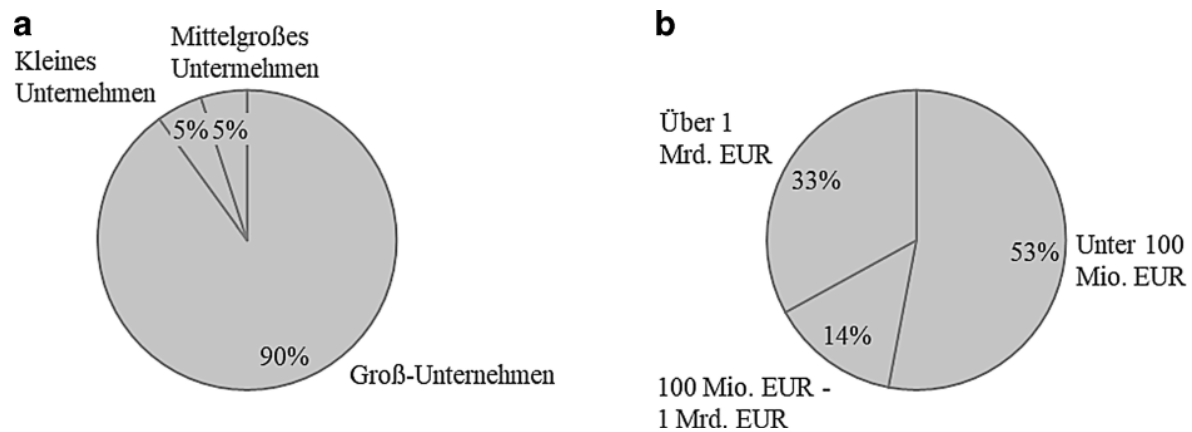

Abb. 2 Unternehmensgröße und Outsourcing-Budget. a Größe des outsourcenden Unternehmens. Klein: Unter 50MA, Umsatz unter 10 Mio. EUR; Mittel: Zw. 50-250MA, Umsatz zw. 10Mio. und 50Mio. EUR; Groß: Über 250 MA, Umsatz über 50Mio. EUR; und b Outsourcing-Budget

\footnotetext{
${ }^{2}$ Der Outsourcing Verband ist ein unabhängiges Netzwerk für Anwender, Berater und Anbieter von IT-
} Geschäftsprozessdienstleistungen mit mehr als 800 Mitgliedern; https://www.outsourcing-verband.org/. 
sich beobachten, dass sowohl IT-Outsourcing-Verträge mit kleinen Volumina von unter 100 Mio. EUR $(37 ; 53 \%)$ als auch sehr große Verträge mit einem Budget von über 1 Mrd. EUR $(23 ; 33 \%)$ für ein Backsourcing in Frage kamen. Abb. 2 fasst die Ergebnisse zusammen.

Die untersuchten Unternehmen kamen aus sehr unterschiedlichen Branchen, unter anderem aus dem Banken- und Versicherungssektor (22; $28 \%$ ), dem Anlagen- und Maschinenbau (22; 28\%), der Technologie- und Medienbranche $(11 ; 14 \%)$ und aus dem Handel- und Konsumgüterbereich $(10 ; 13 \%)$. Weitere Unternehmen kamen aus dem Energie-, dem Chemie- bzw. Pharma- sowie dem Öffentlichen Sektor.

\subsection{Gründe von Backsourcing-Entscheidungen}

Im Folgenden wird vertieft auf das Backsourcing-Phänomen eingegangen. Hierfür wurden deshalb nur diejenigen 51 (64\%) Unternehmen im zugrundeliegenden Datensatz betrachtet, die sich für ein Backsourcing entschieden hatten.

Mit Blick auf die zurückgeholten IT-Services wurden mit $47 \%$ (24) am häufigsten sowohl die Entwicklung von unternehmensspezifischen Applikationen sowie Infrastruktur-Leistungen $(24 ; 47 \%)$ von den befragten Unternehmen backgesourct. Weitere Services, die häufig zurückgeholt wurden, sind Service-Desk-Leistungen (23; $45 \%)$ sowie Endnutzer-Dienste $(21 ; 41 \%)$. Die Tatsache, dass die Applikationsentwicklung eine große Rolle bei IT-Backsourcing-Entscheidungen spielt, verdeutlicht nochmal die eingangs diskutierte, zunehmende Bedeutung der IT für den Unternehmenserfolg, da diese unternehmensspezifischen Applikationen häufig eine große strategische Bedeutung haben. Abb. 3 gibt einen Überblick über die BacksourcingHäufigkeit in Abhängigkeit von der Art des zurückgeholten IT-Services.

Als nächstes wurde die Dauer der zugrunde liegenden Outsourcing-Beziehung betrachtet, bevor es zu einer Backsourcing-Entscheidung kam. Hierbei lässt sich erkennen, dass eine Vielzahl der Verträge bereits nach einer vergleichsweise kurzen Dauer beendet wurden. So wurden $9 \%$ (4) der Verträge in den ersten beiden Jahren und weitere $19 \%$ (8) der Verträge bereits im dritten Jahr beendet. Innerhalb der ersten fünf Jahre wurden kumuliert $60 \%$ (26) aller frühzeitig aufgelösten Verträge bereits aufgelöst. Dagegen liefen $24 \%$ (10) der Verträge über neun Jahre oder länger,

Abb. 3 Art der zurückgeholten IT-Services (Mehrfachnennungen möglich)

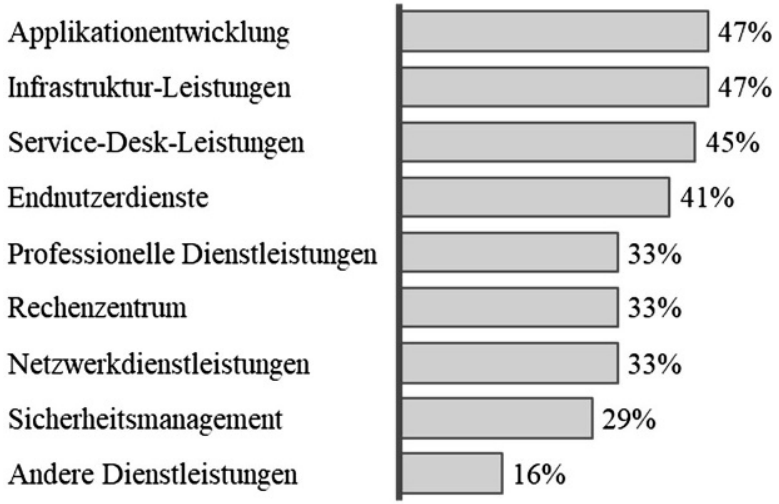


Abb. 4 Dauer der OutsourcingBeziehung vor dem Backsourcing (in Jahren)

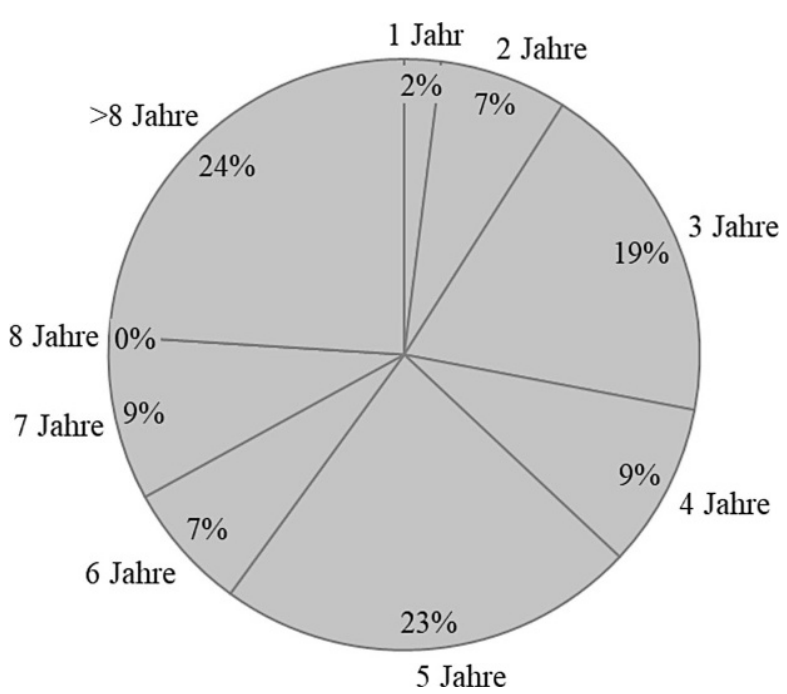

bevor es zu einem Backsourcing kam. Bei diesen Verträgen kam es möglicherweise zu einem Backsourcing nach Ablauf der vereinbarten Vertragslaufzeit und somit nicht zu einer vorzeitigen Vertragsauflösung durch die Backsourcing-Entscheidung. Abb. 4 stellt die Verteilung über die verschiedenen Zeiträume dar.

Betrachtet man die Gründe, warum Unternehmen sich für ein Zurückholen ehemals outgesourcter IT-Services entschieden haben, zeigt sich, dass Unzufriedenheit mit der Qualität der Leistungserbringung mit $65 \%$ (33) der häufigste Grund war. Die zweit- und drittwichtigsten Gründe waren zu hohe Kosten $(25 ; 49 \%)$ und eine Unzufriedenheit mit der Qualität der Beziehung zum Dienstleister $(21 ; 41 \%)$. Weitere erwähnenswerte Gründe waren darüber hinaus noch organisatorische Veränderungen beim Unternehmen $(16 ; 31 \%)$ sowie regulatorische Anforderungen $(7 ; 14 \%)$. Die große Relevanz der Qualität der IT-Services bei der Backsourcing-Entscheidung spiegelt auch die zu Anfang dieses Artikels diskutierte stärkere strategische Bedeutung der IT für den Unternehmenserfolg wider. Abb. 5 fasst die unterschiedlichen Gründe zusammen.

Bei der Beantwortung dieser Frage war die Nennung von mehreren Gründen möglich. Bei den meisten Unternehmen haben im Durchschnitt zwischen 2 und 3 Gründe zu einer Backsourcing-Entscheidung geführt.

Je nach Branche kann der Einfluss verschiedener Gründe schwanken. Dies ließ sich am Beispiel des Banken- und Versicherungssektors gut erkennen, wie ein Zitat aus der Umfrage zeigt:

Im Finanzdienstleistungssektor ist ein Hauptgrund für ein IT-Backsourcing die Einhaltung regulatorischer Anforderungen, wie z. B. Datenschutzrichtlinien.

Das Zitat eines anderen Teilnehmers der Umfrage zeigt beispielhaft, wie die verschiedenen Gründe für ein Out- bzw. Backsourcing in einem Unternehmen zu unterschiedlichen Zeitpunkten im Sourcing-Zyklus betrachtet werden: 
Mangelnde Qualität des IT-Services

$\mathrm{Zu}$ hohe Kosten beim Outsourcing

Mangelnde Beziehungsqualität

Org. Veränderungen beim outsourcenden Unternehmen

Regulatorische Anforderungen

Persönliche Präferenzen der Entscheidungsträger

Ende der Vertragslaufzeit

Org. Veränderungen beim IT-Dienstleister

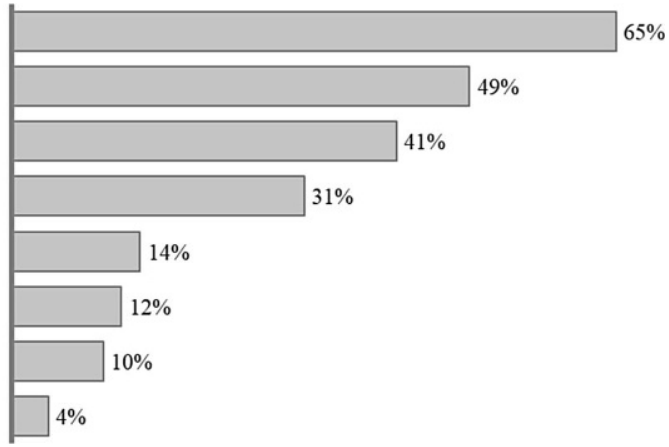

Abb. 5 Gründe für eine Backsourcing-Entscheidung (Mehrfachnennungen möglich)

Während Outsourcing-Entscheidungen oft aus Kostengründen heraus getroffen werden, wird sich für ein Backsourcing meist aufgrund von mangelnder Qualität und schlechter Bereitstellung des Services entschieden. Diese Faktoren werden jedoch bei der ursprünglichen Entscheidungsfindung nicht berücksichtigt, sondern stellen sich häufig erst später als sehr wichtiger Faktor heraus.

\subsection{Resultate der Backsourcing-Entscheidungen}

Neben den Auslösern und Gründen für eine Backsourcing-Entscheidung wurden auch die Ergebnisse einer solchen Entscheidung untersucht. Hier ließ sich insgesamt eine große Zufriedenheit derjenigen Teilnehmer feststellen, die sich für ein Backsourcing entschieden hatten. So zeigt sich, dass $78 \%$ derjenigen Teilnehmer, die IT-Services zurückgeholt hatten, eine hohe Zufriedenheit mit der SourcingEntscheidung im Allgemeinen angaben. Dagegen hatten nur $46 \%$ der Teilnehmer, die sich gegen ein Backsourcing entschieden hatten, eine hohe Zufriedenheit mit der Entscheidung gezeigt. Vergleichbar sind auch die Ergebnisse, wenn man die Qualität des IT-Services bzw. die Kosten der Bereitstellung betrachtet - die Zufriedenheits-

Anteil mit hoher Zufriedenheit ${ }^{\mathrm{a}}$ nach der Entscheidung...

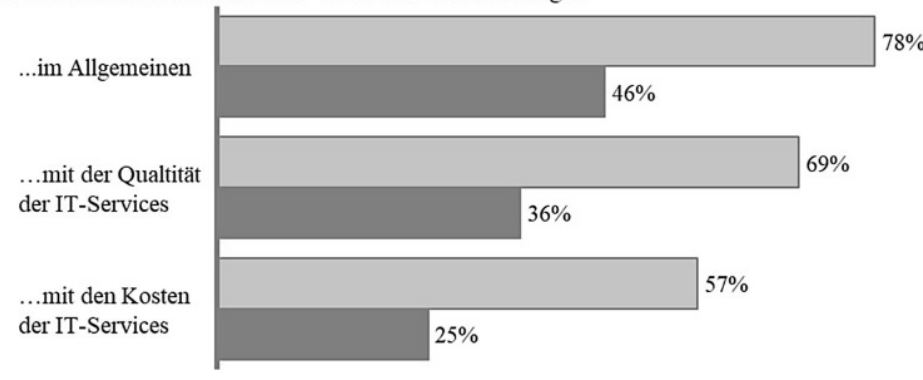

Entscheidung für Backsourcing

Entscheidung gegen Backsourcing

Abb. 6 Zufriedenheit nach der Sourcing-Entscheidung. ${ }^{\text {a}}$ Hohe Zufriedenheit: Antwortmöglichkeiten 5, 6, 7 auf einer Antwortskala von 1 (sehr unzufrieden) bis 7 (sehr zufrieden) 


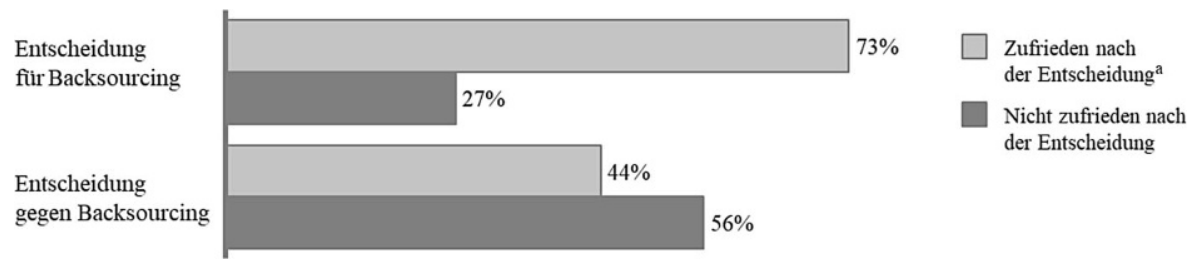

Abb. 7 Zufriedenheit mit der Entscheidung von Unternehmen mit hohen erwarteten Wechselkosten. Anmerkung: Nur Unternehmen berücksichtigt, die hohe Wechselkosten beim Backsourcing erwartet haben. aZufriedenheit: Antwortmöglichkeiten 5, 6, 7 auf einer Antwortskala von 1 (sehr unzufrieden) bis 7 (sehr zufrieden)

werte waren jeweils deutlich höher bei denjenigen Unternehmen, die sich für ein Zurückholen ihrer IT entschieden hatten. Abb. 6 stellt die Ergebnisse dar.

Die Studienergebnisse zeigen, dass - wenngleich sie keine Kausalität begründen - IT-Backsourcing eine relevante Entscheidungsoption in zeitgemäßen SourcingEntscheidungen darstellt, die nicht vernachlässigt werden sollte.

Zudem wurde untersucht, welche Auswirkungen hohe erwartete Wechselkosten (Switching Costs) haben. Beispiele für solche Kosten sind einerseits quantifizierbare Kosten, wie etwa vertraglich definierte Strafzahlungen bei Vertragsauflösung oder Abschreibungen auf Investitionen (Bary und Westner 2018). Andererseits können es auch nicht eindeutig im Vorfeld messbare Kosten sein, wie die entstehende Unsicherheit, eine mögliche Unterbrechung der Serviceerbringung oder (übergangsweise) niedrigere Produktivität. Bei der Auswertung hat sich gezeigt, dass diejenigen Unternehmen, die hohe Wechselkosten erwartet hatten $(n=31 ; 39 \%)$ und sich trotzdem für ein Backsourcing entschieden haben, im Nachhinein deutlich zufriedener waren. Gleichzeitig waren diejenigen Unternehmen, die sich unter anderem aufgrund hoher Wechselkosten gegen ein IT-Backsourcing entschieden haben, nach der Entscheidung am unzufriedensten. Es lässt sich also ableiten, dass Unternehmen froh sind, wenn sie sich von ihren Abhängigkeiten befreit haben, die sich in hohen Wechselkosten manifestieren. Basierend auf diesen Ergebnissen ist es entsprechend zu empfehlen, dass Unternehmen trotz hoher Wechselkosten ein Backsourcing erwägen. Abb. 7 fasst die dargestellten Ergebnisse zusammen.

Die von uns durchgeführte Studie gewährt Einblicke zum Thema IT-Backsourcing in der DACH-Region. Abb. 8 stellt die zentralen Studienergebnissen noch einmal zusammenfassend dar. Es lässt sich feststellen, dass IT-Backsourcing in der Praxis vorkommt und entsprechend für Unternehmen eine sinnvolle Handlungsalternative sein kann, gerade bei bestimmten IT-Services wie der Applikationsentwicklung, bei Infrastruktur-Leistungen oder Service-Desk-Leistungen. Insgesamt zeigt sich eine höhere Zufriedenheit nach der Sourcing-Entscheidung bei denjenigen Unternehmen, die sich für ein IT-Backsourcing entschieden haben - auch bei hohen zu erwartenden Wechselkosten.

In Zukunft werden solche Backsourcing-Vorhaben und Transformationen, wenn sie noch häufiger vorkommen, im Detail zu untersuchen sein, um darauf aufbauend ein geeignetes IT-Backsourcing-Instrumentarium zu entwickeln. Die vorliegende Studie hat in dieser Hinsicht bereits erste Anhaltspunkte für eine erfolgreiche Backsourcing-Durchführung gegeben. 
Backsourcing in der Praxis verbreitet:

- 64\% (51) Unternehmen haben sich für Backsourcing entschieden

Top 3 zurückgeholte IT-Services

- Applikationsentwicklung

- Infrastruktur-Leistungen

- Service-Desk-Leistungen

Top 3 Backsourcing-Gründe

- Mangelnde Qualität des IT-Services

- Zu hohe Kosten beim Outsourcing

- Mangelnde Beziehungsqualität

Ergebnis:

Höhere Zufriedenheitswerte bei den

Unternehmen, die sich für IT-

Backsourcing entschieden haben

- Im Allgemeinen: $78 \%$ vs. $46 \%$

- Mit der Qualität der IT-Services: $69 \%$ vs. $36 \%$

- Mit den Kosten der IT-Services: $57 \%$ vs. $25 \%$

Abb. 8 Zusammenfassung der zentralen Ergebnisse der Umfrage

\section{Handlungsempfehlungen}

Basierend auf bestehenden Forschungserkenntnissen sowie den vorgestellten Studienergebnissen werden nachfolgend Handlungsempfehlungen für IT-Manager in Hinblick auf IT-Sourcing entwickelt. Hierbei lassen sich zwei Dimensionen unterscheiden: allgemeine Empfehlungen für eine zukunftsorientierte IT-Sourcing-Strategie sowie Erfolgsfaktoren für deren erfolgreiche Durchführung.

\subsection{Empfehlungen für eine zukunftsorientierte IT-Sourcing-Strategie}

\subsubsection{Service-spezifische Sourcing-Strategie}

Unternehmen sollten sich von der Vorstellung eines einheitlichen Sourcing-Ansatzes über verschiedene Services und Unternehmensbereiche hinweg lösen und stattdessen eine service-spezifische Sourcing-Strategie verfolgen (Hochstein et al. 2005). So eignet sich nicht jeder IT-Service für die interne Leistungserbringung, da durch ein Outsourcing beispielsweise Effizienzvorteile oder der Zugang zu neuen Technologien genutzt werden können (Benlian und Hess 2011). Umgekehrt sind jedoch manche Services oder Bereiche der IT so wichtig für das Unternehmen, dass sie unbedingt intern erbracht werden sollten, um z. B. eine große Abhängigkeit oder einen Wissensverlust zu verhindern. Intern zu erbringende IT-Services könnten beispielsweise die Systemarchitektur oder die Service-Integration sein (Bary et al. 2018a). Diese Unterscheidung wird durch ein Zitat eines Umfrageteilnehmers verdeutlicht:

Am besten ist es, zwischen Outsourcing und Insourcing entsprechend dem Grad der strategischen Bedeutung und der Kritikalität des betreffenden IT-Services abzuwägen. Für diejenigen Services mit strengen SLAs und hoher Bedeutung für die Organisation würde ich Insourcing empfehlen. Wenn diese Dienste einmal ausgelagert wurden, sollten sie für ein Backsourcing in Betracht gezogen werden.

Entsprechend empfiehlt sich eine service-spezifische Sourcing-Strategie, die die Spezifika individueller Services berücksichtigt und keine „one-size-fits-all“-Lösung 
anstrebt. Beispielsweise könnte die IT-Abteilung unternehmensweite Vorgaben entlang einer Entscheidungsmatrix definieren, mit der die Eignung eines spezifischen Services für verschiedene Sourcing-Formen mittels vordefinierter Kriterien bewertet werden kann.

\subsubsection{Einsatz von verschiedenen, spezialisierten Dienstleistern}

Darüber hinaus ist es zu empfehlen, die Abhängigkeit von einigen wenigen IT-Outsourcing-Dienstleistern zu reduzieren und stattdessen stärker auf eine sogenannte Multisourcing-Strategie zu setzen (Wiener und Saunders 2014). Dadurch lassen sich die spezifischen Vorteile unterschiedlicher Anbieter, wie beispielsweise deren Software oder deren technologisches Know-How, bewusst nutzen (Könning et al. 2018). Darüber hinaus kann so auch der Wettbewerb zwischen den Dienstleistern angeregt werden, um Kostenvorteile zu erreichen (Wiener und Saunders 2014).

Eine Backsourcing-Entscheidung kann strategisch auch dazu genutzt werden, das IT-Sourcing-Setup des Unternehmens neu zu gestalten. Durch ein Backsourcing im ersten Schritt kann ein Vertrag mit einem bestehenden Outsourcing-Dienstleister aufgelöst und die entsprechenden IT-Services können zurück geholt werden, um diese dann im Anschluss partiell wieder an die jeweiligen „,best-of-breed“-Dienstleister outzusourcen. Dies spiegelt sich auch in einem Statement von einem der Umfrageteilnehmer wider:

IT-Backsourcing kann ein Zwischenschritt sein - nach der (frühzeitigen) Terminierung eines IT-Outsourcing-Vertrages - um sich auf eine teilweise NeuVergabe an andere Outsourcing-Partner vorzubereiten, bei der zwischen der Eignung für eine interne oder externe Bereitstellung der Services basierend auf den gewonnenen Erkenntnissen unterschieden wird.

\subsubsection{Langfristige Orientierung der Sourcing-Beziehung}

Drittens empfiehlt sich eine langfristige Orientierung der Sourcing-Beziehung. Unternehmen sollten das Ziel verfolgen, eine partnerschaftliche Beziehung zu ihren Dienstleistern zu unterhalten. Forschung im Bereich IT-Sourcing hat gezeigt, dass eine erfolgreiche Beziehung mit dem Outsourcing-Provider positive Auswirkung auf den Erfolg und die Zufriedenheit mit dem Sourcing-Arrangement hat (Park et al. 2012; Westner und Strahringer 2010).

Gerade im Hinblick auf die zunehmende Verbreitung von agilen Zusammenarbeitsmodellen über IT-Entwicklungsabteilungen hinaus im gesamten Unternehmen und die damit einhergehenden Änderungen in den Zusammenarbeitsformen mit internen und externen Partnern sollte auf eine langfristige, partnerschaftliche Orientierung und eine hohe Beziehungsqualität geachtet werden. Gleichzeitig sollte jedoch die Flexibilität - einer der Hauptvorteile für das outsourcende Unternehmen - nicht verloren gehen und entsprechend eine Abhängigkeit vermieden werden. 


\subsection{Erfolgsfaktoren für eine erfolgreiche Durchführung}

\subsubsection{Fokus auf die Besetzung von Schlüsselstellen}

Ist eine Entscheidung für IT-Backsourcing getroffen worden, sollte frühzeitig der Fokus darauf gesetzt werden, geeignete Personen für die relevanten Schlüsselstellen der neuen bzw. erweiterten IT-Abteilung zu finden. Schlüsselstellen sind beispielsweise IT-Architekten, Product Owner oder Cybersecurity-Spezialisten (White 2018). Eine Möglichkeit besteht auch darin, gezielt Mitarbeiter des Outsourcing-Anbieters zu übernehmen, da diese bereits wichtiges, unternehmensspezifisches Wissen besitzen (Salge 2015). Einer der Teilnehmer der Umfrage berichtet dazu wie folgt:

Wir hätten sehr davon profitiert, einigen der herausragenden Ressourcen unseres Dienstleisters einen Job anzubieten, um sie bei uns im Unternehmen zu integrieren.

Sind diese Schlüsselstellen besetzt - z. B. durch Übernahme vom Dienstleister, durch einen internen Arbeitsplatzwechsel oder durch Neueinstellungen vom Arbeitsmarkt - lässt sich um diese Stellen herum die weitere Organisationseinheit entsprechend aufbauen. Wichtig ist es, dass diese Schlüsselpositionen möglichst früh - vor dem Auslaufen des Outsourcing-Vertrages - besetzt sind, um frühzeitig mit dem Aufbau der entsprechenden internen IT-Organisationseinheit beginnen zu können und so einen reibungslosen Übergang der IT-Serviceerbringungen zu gewährleisten.

\subsubsection{Lückenloser Wissenstransfer}

Des Weiteren sollte auf einen möglichst lückenlosen Wissenstransfer geachtet werden (Cha et al. 2008). Während beim Outsourcing der neue IT-Dienstleister ein großes Interesse an einem reibungslosen Übergang hat, wird das Unternehmen beim Backsourcing häufig auf weniger Unterstützung des scheidenden Dienstleisters zählen können. Entsprechend wichtig ist es, auf eine gute Dokumentation und Übergabe zu achten. Beispielsweise könnte ein Teil des neu aufgebauten Teams, wie die bereits diskutieren Schlüsselstellen, für die Zeit der Übergabe „,co-located“ mit dem IT-Dienstleister arbeiten, um so in kurzer Zeit möglichst viel Wissen zu übernehmen (Benaroch et al. 2010). Einer der Studienteilnehmer beschrieb das Vorgehen in seinem Unternehmen wie folgt:

Es sieht so aus, als ob der Trend Richtung co-located Teams geht. Wir haben externe IT-Ressourcen von Indien nach Großbritannien backgesourct, damit die Kollegen nebeneinander sitzen können und wir so die Schnelligkeit und Kommunikation verbessern können!

Zusätzlich kann es für das Unternehmen sinnvoll sein, mit dem scheidenden Outsourcing-Dienstleister eine Übergangsregelung zu finden, in der beispielsweise einzelne Ressourcen noch als offizielle Kontaktpersonen ansprechbar bleiben oder den Aufbau der IT-Abteilung unterstützen. 
Tab. 1 Zusammenfassung der Handlungsempfehlungen

\begin{tabular}{ll}
\hline $\begin{array}{l}\text { Empfehlungen für eine zukunftsorientierte IT- } \\
\text { Sourcing-Strategie }\end{array}$ & $\begin{array}{l}\text { Erfolgsfaktoren für eine erfolgreiche Backsour- } \\
\text { cing-Durchführung }\end{array}$ \\
\hline Service-spezifische Sourcing-Strategie & $\begin{array}{l}\text { Fokus auf die Besetzung von Schlüsselstellen } \\
\text { Lückenloser Wissenstransfer }\end{array}$ \\
$\begin{array}{l}\text { Einsatz von verschiedenen, spezialisierten Dienst- } \\
\text { leistern }\end{array}$ & Proaktive interne und externe Kommunikation \\
\hline Langfristige Orientierung der Sourcing-Beziehung
\end{tabular}

\subsubsection{Proaktive interne und externe Kommunikation}

Außerdem sollten Unternehmen auf eine umfassende Kommunikation der neuen Strategie und der sich dadurch ergebenden Änderungen an die verschiedenen Stakeholder wie z.B. Mitarbeiter, Kunden oder andere IT-Dienstleister achten (Bhagwatwar et al. 2011). Je nach Größe des zurückgeholten Outsourcing-Volumens ist es wahrscheinlich, dass es während der Übergangszeit zu gewissen Einschränkungen, beispielsweise bei der Verfügbarkeit einzelner Systeme, kommt.

Entsprechend hilft es, frühzeitig möglicherweise betroffene Anwender zu analysieren, diese auf kurzfristige Beeinträchtigungen vorzubereiten und ihnen die Notwendigkeit und zukünftige Verbesserungen transparent aufzuzeigen. Dadurch lässt sich das Verständnis der Betroffenen erhöhen und gleichzeitig kann die Kommunikationskampagne genutzt werden, um das Unternehmen im Gesamten als positiv und zukunftsorientiert darzustellen.

Abschließend fasst Tab. 1 die Handlungsempfehlungen für eine zukunftsorientierte IT-Sourcing-Strategie und für eine erfolgreiche Backsourcing-Durchführung in einer Übersicht zusammen.

\section{Zusammenfassung und Ausblick}

Die von uns durchgeführte Studie zum Thema „Einflussfaktoren von IT-Backsourcing-Entscheidungen" gewährt Einblicke zum Thema IT-Backsourcing in der DACH-Region. Sie ist eine der ersten Studien, die dieses Phänomen spezifisch in der DACH-Region empirisch betrachtet.

IT-Backsourcing wird von Unternehmen in unterschiedlichen Branchen durchgeführt, dafür kommen verschiedene IT-Services in Betracht. Hauptgründe für ein ITBacksourcing sind mangelnde Qualität, hohe Kosten sowie eine schlechte Beziehung zum IT-Dienstleister. Die Unternehmen, die sich für ein Backsourcing entschieden haben, sind nach der Entscheidung insgesamt zufriedener als die Unternehmen, die in einer Outsourcing-Beziehung verblieben sind. Diese Ergebnisse sollten hinsichtlich ihrer tieferen Ursachen und spezifischer Rahmenbedingungen, z. B. in Einzelfallstudien, weiter untersucht werden. Zudem könnte eine tiefgängige, zugleich aber auch breite Untersuchung von Backsourcing-Vorgängen dazu beitragen, ein ausgereiftes Backsourcing-Instrumentarium aufzubauen. Im Kontext der nach wie vor anhaltenden digitalen Transformation vieler Unternehmen könnte sich dies zu einem wichtigen Transformationsbaustein entwickeln. 
Funding Open Access funding provided by Projekt DEAL.

Open Access Dieser Artikel wird unter der Creative Commons Namensnennung 4.0 International Lizenz veröffentlicht, welche die Nutzung, Vervielfältigung, Bearbeitung, Verbreitung und Wiedergabe in jeglichem Medium und Format erlaubt, sofern Sie den/die ursprünglichen Autor(en) und die Quelle ordnungsgemäß nennen, einen Link zur Creative Commons Lizenz beifügen und angeben, ob Änderungen vorgenommen wurden.

Die in diesem Artikel enthaltenen Bilder und sonstiges Drittmaterial unterliegen ebenfalls der genannten Creative Commons Lizenz, sofern sich aus der Abbildungslegende nichts anderes ergibt. Sofern das betreffende Material nicht unter der genannten Creative Commons Lizenz steht und die betreffende Handlung nicht nach gesetzlichen Vorschriften erlaubt ist, ist für die oben aufgeführten Weiterverwendungen des Materials die Einwilligung des jeweiligen Rechteinhabers einzuholen.

Weitere Details zur Lizenz entnehmen Sie bitte der Lizenzinformation auf http://creativecommons.org/ licenses/by/4.0/deed.de.

\section{Literatur}

v Bary B (2018) How to bring IT home: developing a common terminology to compare cases of IS backsourcing. In: AMCIS 2018

v Bary B, Westner M (2018) Information systems backsourcing: a literature review. J Inf Technol Manag 1(29):62-78

v Bary B, Westner M, Strahringer S (2018a) Adding experts' perceptions to complement existing research on information systems backsourcing. Int J Inf Syst Proj Manag 6(4):17-35

v Bary B, Westner M, Strahringer S (2018b) Do researchers investigate what practitioners deem relevant?: gaps between research and practice in the field of information systems backsourcing. In: CBI 2018, S 40-49

Benaroch M, Dai Q, Kauffman RJ (2010) Should we go our own way?: Backsourcing flexibility in IT services contracts. J Manag Inf Syst 26(4):317-358

Benlian A, Hess T (2011) Opportunities and risks of software-as-a-service: findings from a survey of IT executives. Decis Support Syst 52(1):232-246

Bhagwatwar A, Hackney R, Desouza KC (2011) Considerations for information systems "Backsourcing": a framework for knowledge re-integration. Inf Syst Manag 28(2):165-173

Butler N, Slack F, Walton J (2011) IS/IT backsourcing: a case of outsourcing in reverse? In: HICSS 2011

Cha HS, Pingry DE, Thatcher ME (2008) Managing the knowledge supply chain: an organizational learning model of information technology outsourcing. MISQ 32(2):281-306

Dibbern J, Goles T, Hirschheim R, Jayatilaka B (2004) Information systems outsourcing: a survey and analysis of the literature. SIGMIS Database 35(4):6-102

Heeg T (2018) Unzufriedene Kunden: Die Telekom verliert einen Millionenauftrag. https://www.faz.net/ aktuell/wirtschaft/diginomics/telekom-t-systems-und-thyssen-krupp-kuendigen-vertrag-15428310. html. Zugegriffen: 20. Mai 2019

Hirschheim R, Lacity M (1998) Backsourcing: an emerging trend? http://www.outsourcing-center.com/ 1998-09-backsourcing-an-emerging-trend-article-38943.html. Zugegriffen: 15. Jan. 2018

Hochstein A, Tamm G, Brenner W (2005) Service oriented IT management: benefit, cost and success factors. In: ECIS 2005

Karabasz I, Wocher M (2018) Großauftrag verloren: Rückschlag für T-Systems. http://www.handelsblatt. com/20916242-all.html. Zugegriffen: 5. Apr. 2018

Ketterer H, Rehberg B, Schmid C, Kleine D (2016) The end of two-speed IT. https://www.bcg.com/de-de/ publications/2016/software-agile-digital-transformation-end-of-two-speed-it.aspx. Zugegriffen: 7. Mai 2019

Könning M, Westner M, Strahringer S (2018) Multisourcing on the rise: results from an analysis of more than 1,000 IT outsourcing deals in the ASG region. In: MKWI 2018, S 1813-1824

Lacity MC, Willcocks LP (2000) Relationships in IT outsourcing: a stakeholder perspective. In: Zmud RW (Hrsg) Framing the domains of IT management: projecting the future through the past. Pinnaflex Educational Resources, Inc, Cincinnati, S 355-384 
Nickel O (2018) T-Systems und Thyssenkrupp: 700-Millionen-Euro-Vertrag für IT-Migration verloren - Golem.de. https://www.golem.de/news/t-systems-und-thyssenkrupp-700-millionen-euro-vertragfuer-it-migration-verloren-1802-132532.html. Zugegriffen: 20. Mai 2019

Nujen B, Halse L, Solli-Saether H (2015) Backsourcing and knowledge re-integration: a case study. In: IFIP 2015, S 191-198

Oshri I, Kotlarsky J, Willcocks L (Hrsg) (2015) The handbook of global outsourcing and offshoring, 3. Aufl. Palgrave Macmillan, Basingstoke, United Kingdom

Park J, Lee J, Lee H, Truex D (2012) Exploring the impact of communication effectiveness on service quality, trust and relationship commitment in IT services. Int J Inf Manage 32(5):459-468

Salge C (2015) Pulling the outside in: a transactional cost perspective on IT insourcing. In: AMCIS 2015

Strasser A, Westner M (2015) Information systems offshoring: results of a systematic literature review. J Inf Technol Manag 26(2):70-142

Thakur-Wernz P (2019) A typology of backsourcing: short-run total costs and internal capabilities for reinternalization. J Glob Oper Strateg Sourc 12(1):42-61

Westner M, Strahringer S (2010) Determinants of success in IS offshoring projects: results from an empirical study of German companies. Inf Manag 47(5-6):291-299

White SK (2018) The 13 most in-demand tech jobs for 2019-and how to hire for them. https://www.cio. com/article/3235944/hiring-the-most-in-demand-tech-jobs-for-2018.html. Zugegriffen: 8. Mai 2019

Wiener M, Saunders C (2014) Forced coopetition in IT multi-sourcing. J Strateg Inf Syst 23(3):210-225 